\title{
Periodic solutions for prescribed mean curvature Rayleigh equation with a deviating argument
}

\author{
Jin $\mathrm{Li}^{1,2^{*}}$, Jianlin $\mathrm{LuO}^{1}$ and Yun Cai ${ }^{2}$
}

\section{"Correspondence:}

lijin7912@gmail.com

${ }^{1}$ College of Science, Jiujiang

University, Jiujiang, 332005, China

${ }^{2}$ School of Mathematical Sciences, Capital Normal University, Beijing, 100048, China

\begin{abstract}
By using Mawhin's continuation theorem in the coincidence degree theory, some criteria for guaranteeing the existence of periodic solutions for prescribed mean curvature Rayleigh equation with a deviating argument are provided.
\end{abstract}

Keywords: prescribed mean curvature Rayleigh equation; periodic solutions; Leray-Schauder degree

\section{Introduction}

Consider the prescribed mean curvature Rayleigh equation

$$
\left(\frac{x^{\prime}}{\sqrt{1+x^{\prime 2}}}\right)^{\prime}+f\left(t, x^{\prime}(t)\right)+g(t, x(t-\tau(t)))=e(t)
$$

where $\tau, e \in C(\mathbb{R}, \mathbb{R})$ are $T$-periodic, and $f, g \in C(\mathbb{R} \times \mathbb{R}, \mathbb{R})$ are $T$-periodic in the first argument, $T>0$ is a constant.

In recent years, there are many results on the existence of periodic solutions for various types of delay differential equation with deviating arguments, especially for the Liénard equation and Rayleigh equation (see [1-11]). Now as the prescribed mean curvature $\left(\frac{x^{\prime}(t)}{\sqrt{1+x^{\prime 2}(t)}}\right)^{\prime}$ of a function $x(t)$ frequently appears in different geometry and physics (see [1214]), it is interesting to try to consider the existence of periodic solutions of prescribed mean curvature equations. However, to our best knowledge, the studies of delay equations with prescribed mean curvature is relatively infrequent. The main difficulty lies in the nonlinear term $\left(\frac{x^{\prime}(t)}{\sqrt{1+x / 2(t)}}\right)^{\prime}$, the existence of which obstructs the usual method of finding a priori bounds for delay Liénard or Rayleigh equations from working. In [15], Feng discussed a delay prescribed mean curvature Liénard equation of the form

$$
\left(\frac{x^{\prime}}{\sqrt{1+x^{\prime 2}}}\right)^{\prime}+f(x(t)) x^{\prime}(t)+g(t, x(t-\tau(t)))=e(t),
$$

estimated a priori bounds by eliminating the nonlinear term $\left(\frac{x^{\prime}(t)}{\sqrt{1+x^{\prime 2}(t)}}\right)^{\prime}$, and established sufficient conditions on the existence of periodic solutions for (1.2) by using Mawhin's continuation theorem.

The conditions imposed on $f(x)$ and $g(t, x)$ in [15] were such as: 
$\left(C_{1}\right)$ There exists $\gamma>0$ satisfies $|f(x)| \geq \gamma$.

$\left(C_{2}\right)$ There exists $l>0$ such that $\left|g\left(t, x_{1}\right)-g\left(t, x_{2}\right)\right| \leq l\left|x_{1}-x_{2}\right|, \forall t \in \mathbb{R}, x_{1}, x_{2} \in \mathbb{R}$.

It is not difficult to see that the condition $\left(\mathrm{C}_{1}\right)$ is strong. It is natural to relax the conditions $\left(C_{1}\right)$ and $\left(C_{2}\right)$. Our purpose is studying the more general equation (1.1) under the more weaker conditions.

The rest of the paper is organized as follows. In Section 2, we shall state and prove some basic lemmas. In Section 3, we shall prove the main result. An example will be given to show the applications of our main result in the final section.

\section{Preliminaries}

In this section, we first recall Mawhin's continuation theorem, which our study is based upon.

Let $X$ and $Y$ be real Banach spaces and $L: X \supset \operatorname{Dom} L \rightarrow Y$ be a linear operator. $L$ is said to be a Fredholm operator with index zero provided that

(i) $\operatorname{Im} L$ is closed subset of $Y$,

(ii) $\operatorname{dim} \operatorname{ker} L=\operatorname{codim} \operatorname{Im} L<+\infty$.

Set $X=\operatorname{ker} L \oplus X_{1}, Y=\operatorname{Im} L \oplus Y_{1}$. Let $P: X \rightarrow \operatorname{ker} L$ and $Q: Y \rightarrow Y_{1}$ be the nature projections. It is easy to see that $\operatorname{ker} L \cap\left(\operatorname{Dom} L \cap X_{1}\right)=0$. Thus, the restriction $L_{p}:=\left.L\right|_{\operatorname{Dom} L \cap X_{1}}$ is invertible. We denote by $k$ the inverse of $L_{p}$.

Let $\Omega$ be a open bounded subset of $X$ with $\operatorname{Dom} L \cap \Omega \neq \phi$. A map $N: \bar{\Omega} \rightarrow Y$ is said to be $L$-compact in $\bar{\Omega}$ if $Q N: \bar{\Omega} \rightarrow Y$ and $k(I-Q) N: \bar{\Omega} \rightarrow X$ are compact.

The following lemma due to Mawhin [16] is fundamental to prove our main result.

Lemma 2.1 Let L be a Fredholm operator of index zero and Let $N$ be L-compact on $\bar{\Omega}$. If the following conditions hold:

$\left(\mathrm{h}_{1}\right) \quad L x \neq \lambda N x, \forall(x, \lambda) \in[(D(L) \backslash \operatorname{Ker} L) \cap \partial \Omega] \times(0,1)$;

$\left(\mathrm{h}_{2}\right) \quad N x \notin \operatorname{Im} L, \forall x \in \operatorname{Ker} L \cap \partial \Omega$;

$\left(\mathrm{h}_{3}\right) \operatorname{deg}\left(\left.J Q N\right|_{\operatorname{Ker} L}, \Omega \cap \operatorname{Ker} L, 0\right) \neq 0$.

Then $L x=N x$ has at least one solution in $D(L) \cap \bar{\Omega}$.

The following lemmas is useful in the proof of our main result.

Lemma 2.2 ([17]) Let $s \in C(\mathbb{R}, \mathbb{R})$ with $s(t) \in[0, T], \forall t \in \mathbb{R}$. Suppose $p \in(1,+\infty)$, $\alpha=$ $\max _{t \in[0, T]} s(t)$ and $u \in C^{1}(\mathbb{R}, \mathbb{R})$ with $u(t+\omega)=u(t)$. Then

$$
\int_{0}^{T}|u(t)-u(t-s(t))|^{p} d t \leq \alpha^{p} \int_{0}^{T}\left|u^{\prime}(t)\right|^{p} d t .
$$

Lemma 2.3 ([18]) If $x \in C_{T}^{1}(\mathbb{R}, \mathbb{R})$ and $\int_{0}^{T} x(t) d t=0$, then

$$
\int_{0}^{T}|x(t)|^{2} d t \leq\left(T^{2} / 4 \pi^{2}\right) \int_{0}^{T}\left|x^{\prime}(t)\right|^{2} d t
$$

(Wirtinger inequality) and

$$
|x|_{\infty}^{2} \leq(T / 12) \int_{0}^{T}\left|x^{\prime}(t)\right|^{2} d t
$$

(Sobolev inequality). 
Lemma 2.4 ([18]) Suppose $x(t) \in C^{1}[0, T]$, and $x(0)=x(T)=0$. Then

$$
\int_{0}^{T}|x(t)|^{2} d t \leq \frac{T^{2}}{\pi^{2}} \int_{0}^{T}\left|x^{\prime}(t)\right|^{2} d t
$$

Lemma 2.5 Assume that $x(t) \in C^{1}[0, T]$, and $x(0)=x(T)=0$. Then

$$
|x(t)| \leq \frac{1}{2} \sqrt{T}\left(\int_{0}^{T}\left|x^{\prime}(t)\right|^{2} d t\right)^{2}, \quad \forall t \in[0, T]
$$

Proof It is easy to see that

$$
|x(t)|=\left|x(0)+\int_{0}^{t} x^{\prime}(s) d s\right| \leq \int_{0}^{t}\left|x^{\prime}(s)\right| d s, \quad \forall t \in[0, T]
$$

and

$$
|x(t)|=\left|x(T)-\int_{t}^{T} x^{\prime}(s) d s\right| \leq \int_{t}^{T}\left|x^{\prime}(s)\right| d s, \quad \forall t \in[0, T] .
$$

Combining the above inequalities and using Hölder's inequality,

$$
|x(t)| \leq \frac{1}{2} \int_{0}^{T}\left|x^{\prime}(s)\right| d s \leq \frac{1}{2} \sqrt{T}\left(\int_{0}^{T}\left|x^{\prime}(t)\right|^{2} d t\right)^{2}, \quad \forall t \in[0, T] .
$$

The proof is completed.

In order to apply Mawhin's continuation theorem to study the existence of $T$-periodic solution of Equation (1.1), we rewrite (1.1) as

$$
\left\{\begin{array}{l}
x^{\prime}(t)=\frac{y(t)}{\sqrt{1-y^{2}(t)}}, \\
y^{\prime}(t)=-f\left(t, \frac{y(t)}{\sqrt{1-y^{2}(t)}}\right)-g(t, x(t-\tau(t)))+e(t) .
\end{array}\right.
$$

Obviously, if $z(t)=(x(t), y(t))^{\top}$ is a $T$-periodic solution of (2.1), then $x(t)$ must be a $T$ periodic solution of (1.1). Hence, the problem of finding a $T$-periodic solution of (1.1) reduces to finding one of (2.1).

Now, we set

$$
X=Y=\left\{z: z(t)=(x(t), y(t))^{\top} \in C\left(\mathbb{R}^{1}, \mathbb{R}^{2}\right), z(t)=z(t+T)\right\},
$$

with the norm $\|z\|=\max \left\{\|x\|_{\infty},\|y\|_{\infty}\right\}$, where

$$
\|x\|_{\infty}=\max _{t \in[0, T]}|x(t)|, \quad\|y\|_{\infty}=\max _{t \in[0, T]}|y(t)| .
$$

Clearly, $X$ and $Y$ are Banach spaces. Meanwhile, let

$$
L: X \supset \operatorname{Dom} L \rightarrow Y, \quad L z=z^{\prime}=\left(x^{\prime}(t), y^{\prime}(t)\right)^{\top},
$$


where

$$
\operatorname{Dom} L=\left\{z: z=(x(t), y(t))^{\top} \in C^{1}\left(\mathbb{R}, \mathbb{R}^{2}\right), z(t)=z(t+T)\right\}
$$

Define a nonlinear operator $N: X \rightarrow Y$ by

$$
N z=\left(\begin{array}{c}
\frac{y(t)}{\sqrt{1-y^{2}(t)}} \\
-f\left(t, \frac{y(t)}{\sqrt{1-y^{2}(t)}}\right)-g\left(t, x_{1}(t-\tau(t))\right)+e(t)
\end{array}\right) .
$$

Then the problem (2.1) can be written to $L z=N z$.

It is easy to see that $\operatorname{ker} L=\mathbb{R}^{2}$ and $\operatorname{Im} L=\left\{u \in Y: \int_{0}^{T} u(s) d s=0\right\}$. So, $L$ is a Fredholm operator with index zero.

Let $P: X \rightarrow \operatorname{ker} L$ and $Q: Y \rightarrow \operatorname{Im} Q$ be defined by

$$
P z=\frac{1}{T} \int_{0}^{T} z(s) d s, \quad Q u=\frac{1}{T} \int_{0}^{T} u(s) d s
$$

and denote by $k$ the inverse of $\left.L\right|_{\operatorname{ker} P \cap \operatorname{Dom} L}$. Then $\operatorname{ker} L=\operatorname{Im} Q=\mathbb{R}^{2}$ and

$$
k u(t)=\int_{0}^{T} G(t, s) u(s) d s
$$

where

$$
G(t, s)= \begin{cases}\frac{s}{T}, & 0 \leq s<t \leq T, \\ \frac{s-T}{T}, & 0 \leq t \leq s \leq T .\end{cases}
$$

It follows from (2.2) that $N$ is $L$-compact on $\bar{\Omega}$, where $\Omega$ is an open, bounded subset of $X$.

\section{Main results}

In this section, we will state and prove our main results.

We first give the following assumptions:

(H1) $f(t, 0)=0$, and $x f(t, x) \geq 0$ (or $x f(t, x) \leq 0), \forall t \in \mathbb{R}$.

(H2) $x g(t, x) \geq 0$ (or $x g(t, x) \leq 0), \forall t \in \mathbb{R}$, and $|g(t, x)|>\|e\|_{\infty}$ for $|x|>d$.

(H3) There exists $r_{1}, r_{2}, r_{3}>0$ such that

$$
r_{1} \leq \liminf _{|x| \rightarrow \infty} \frac{|f(t, x)|}{|x|} \leq \limsup _{|x| \rightarrow \infty} \frac{|f(t, x)|}{|x|} \leq r_{2}, \quad \text { uniformly in } t \in \mathbb{R}
$$

and

$$
\limsup _{|x| \rightarrow \infty} \frac{|g(t, x)|}{|x|} \leq r_{3}, \quad \text { uniformly in } t \in \mathbb{R} .
$$

(H4) There exists an integer $m$ such that $0 \leq \tau(t)-m T \leq T$, and

$$
\alpha:=\|\tau(t)-m T\|_{\infty} \leq T .
$$


Theorem 3.1 Assume (H1)-(H4) hold. Then Equation (1.1) has at least one T-periodic solution provided

$$
\frac{r_{3}}{r_{1}}<\max \left\{\frac{2}{T}, \frac{1}{\alpha+\frac{T}{\pi}}\right\}
$$

Proof Consider the operator equation

$$
L z=\lambda N z, \quad \forall \lambda \in(0,1) .
$$

Let $\Omega_{1}=\{z \in X: L z=\lambda N z, \lambda \in(0,1)\}$. If $z(t)=(x(t), y(t))^{\top} \in \Omega_{1}$, we have

$$
\left\{\begin{array}{l}
x^{\prime}(t)=\lambda \frac{y(t)}{\sqrt{1-y^{2}(t)}} \\
y^{\prime}(t)=-\lambda f\left(t, \frac{y(t)}{\sqrt{1-y^{2}(t)}}\right)-\lambda g(t, x(t-\tau(t)))+\lambda e(t),
\end{array}\right.
$$

It follows from the first equation of (3.3) that

$$
y(t)=\frac{\frac{1}{\lambda} x^{\prime}(t)}{\sqrt{1+\frac{1}{\lambda^{2}} x^{\prime 2}(t)}} .
$$

Then (3.3) can be written to

$$
\left(\frac{\frac{1}{\lambda} x^{\prime}(t)}{\sqrt{1+\frac{1}{\lambda^{2}} x^{\prime 2}(t)}}\right)^{\prime}=-\lambda f\left(t, \frac{1}{\lambda} x^{\prime}(t)\right)-\lambda g(t, x(t-\tau(t)))+\lambda e(t) .
$$

Integrating the first equation of (3.3) from 0 to $T$, we have

$$
\int_{0}^{T} \frac{y(t)}{\sqrt{1-y^{2}(t)}} d t=0
$$

Then there exist $t_{1}, t_{2} \in[0, T]$, such that

$$
y\left(t_{1}\right) \geq 0, \quad y\left(t_{2}\right) \leq 0 .
$$

Assume that $t_{3}, t_{4} \in[0, T]$ are the maximum and minimum points, respectively. Then

$$
y\left(t_{3}\right) \geq 0, \quad y^{\prime}\left(t_{3}\right)=0,
$$

and

$$
y\left(t_{4}\right) \leq 0, \quad y^{\prime}\left(t_{4}\right)=0 .
$$

It follows from the second equation of (3.3) that

$$
0=y^{\prime}\left(t_{3}\right)=-\lambda f\left(t_{3}, \frac{y\left(t_{3}\right)}{\sqrt{1-y^{2}\left(t_{3}\right)}}\right)-\lambda g\left(t_{3}, x\left(t_{3}-\tau\left(t_{3}\right)\right)\right)+\lambda e\left(t_{3}\right) .
$$


From (H1) and (H2), without loss of generality, we can assume that $x f(t, x) \geq 0$ and $x g(t, x) \geq 0, \forall x \in \mathbb{R}$. Then

$$
g\left(t_{3}, x\left(t_{3}-\tau\left(t_{3}\right)\right)\right) \leq e\left(t_{3}\right) \leq\|e\|_{\infty} .
$$

If $x\left(t_{3}-\tau\left(t_{3}\right)\right)>d$, then $g\left(t_{3}, x\left(t_{3}-\tau\left(t_{3}\right)\right)\right)>\|e\|_{\infty}$, which is a contradiction. It follows that

$$
x\left(t_{3}-\tau\left(t_{3}\right)\right) \leq d .
$$

Similarly, from (3.6), we have

$$
x\left(t_{4}-\tau\left(t_{4}\right)\right) \geq-d .
$$

Combining the above, we know that there exists a point $\xi \in[0, T]$ such that

$$
|x(\xi-\tau(\xi))| \leq d
$$

Note that there exist $k \in \mathbb{Z}$ and $t^{*} \in[0, T]$ such that $\xi-\tau(\xi)=k T+t^{*}$. Then we get

$$
\left|x\left(t^{*}\right)\right| \leq d
$$

By Lemma 2.4, we obtain

$$
\begin{aligned}
|x(t)| & \leq\left|x\left(t^{*}\right)\right|+\left|x(t)-x\left(t^{*}\right)\right| \\
& \leq\left|x\left(t^{*}\right)\right|+\frac{1}{2} \sqrt{T}\left(\int_{t^{*}}^{T^{*}+T}\left|x^{\prime}(t)\right|^{2} d t\right)^{\frac{1}{2}} \\
& =d+\frac{1}{2} \sqrt{T}\left\|x^{\prime}\right\|_{2} .
\end{aligned}
$$

Hence,

$$
\|x\|_{\infty} \leq d+\frac{1}{2} \sqrt{T}\left\|x^{\prime}\right\|_{2} .
$$

Meanwhile, by Lemma 2.3, we have

$$
\begin{aligned}
\|x\|_{2} & =\left(\int_{0}^{T}|x(t)|^{2} d t\right)^{\frac{1}{2}} \\
& =\left(\int_{0}^{T}\left|x\left(t+t^{*}\right)\right|^{2} d t\right)^{\frac{1}{2}} \\
& =\left(\int_{0}^{T}\left|x\left(t+t^{*}\right)-x\left(t^{*}\right)+x\left(t^{*}\right)\right|^{2} d t\right)^{\frac{1}{2}} \\
& \leq\left(\int_{0}^{T}\left(\left|x\left(t+t^{*}\right)-x\left(t^{*}\right)\right|+d\right)^{2} d t\right)^{\frac{1}{2}} \\
& =\left(\int_{0}^{T}\left(\left|x\left(t+t^{*}\right)-x\left(t^{*}\right)\right|^{2}+2 d\left|x\left(t+t^{*}\right)-x\left(t^{*}\right)\right|+d^{2}\right) d t\right)^{\frac{1}{2}}
\end{aligned}
$$




$$
\begin{aligned}
& \leq\left(\frac{T^{2}}{\pi^{2}} \int_{0}^{T}\left|x^{\prime}(t)\right|^{2} d t+2 d \sqrt{T} \frac{T}{\pi}\left(\int_{0}^{T}\left|x^{\prime}(t)\right|^{2} d t\right)^{\frac{1}{2}}+d^{2} T\right)^{\frac{1}{2}} \\
& =\frac{T}{\pi}\left\|x^{\prime}\right\|_{2}+d \sqrt{T} .
\end{aligned}
$$

From (3.1), we have $\frac{T}{2} r_{3}<r_{1}$, or $r_{3}\left(\alpha+\frac{T}{\pi}\right)<r_{1}$. Then there exists $\varepsilon>0$ such that

$$
\frac{T}{2}\left(r_{3}+\varepsilon\right)<r_{1}-\varepsilon
$$

or

$$
\left(r_{3}+\varepsilon\right)\left(\alpha+\frac{T}{\pi}\right)<\left(r_{1}-\varepsilon\right) .
$$

For such a $\varepsilon>0$, it follows from (H3), there exist $h_{1}, h_{2} \geq 0$ such that

$$
\left(r_{1}-\varepsilon\right)|x|-h_{1} \leq|f(t, x)| \leq\left(r_{2}+\varepsilon\right)|x|+h_{1}, \quad \forall t, x \in \mathbb{R},
$$

and

$$
|g(t, x)| \leq\left(r_{3}+\varepsilon\right)|x|+h_{2}, \quad \forall t, x \in \mathbb{R}
$$

Multiplying $x^{\prime}(t)$ and (3.4) and integrating from 0 to $T$, we get

$$
\lambda \int_{0}^{T} f\left(t, \frac{1}{\lambda} x^{\prime}(t)\right) x^{\prime}(t) d t+\lambda \int_{0}^{T} g(t, x(t-\tau(t))) x^{\prime}(t) d t=\lambda \int_{0}^{T} e(t) x^{\prime}(t) d t .
$$

It follows from (H1) and (3.13) that

$$
\begin{aligned}
\left|\lambda \int_{0}^{T} f\left(t, \frac{1}{\lambda} x^{\prime}(t)\right) x^{\prime}(t) d t\right| & =\lambda \int_{0}^{T}\left|f\left(t, \frac{1}{\lambda} x^{\prime}(t)\right) x^{\prime}(t)\right| d t \\
& \geq\left(r_{1}-\varepsilon\right) \int_{0}^{T}\left|x^{\prime}(t)\right|^{2} d t-\lambda \int_{0}^{T} h_{1}\left|x^{\prime}(t)\right| d t .
\end{aligned}
$$

Substituting (3.16) into (3.15) and from (3.14), we have

$$
\begin{aligned}
\left(r_{1}-\varepsilon\right) & \int_{0}^{T}\left|x^{\prime}(t)\right|^{2} d t \\
\leq & \lambda\left|\int_{0}^{T} g(t, x(t-\tau(t))) x^{\prime}(t) d t\right|+\lambda\left|\int_{0}^{T} e(t) x^{\prime}(t) d t\right| \\
& +\lambda\left|\int_{0}^{T} h_{1} x^{\prime}(t) d t\right| \\
\leq & \int_{0}^{T}|g(t, x(t-\tau(t)))|\left|x^{\prime}(t)\right| d t+\left(\|e\|_{\infty}+h_{1}\right) \int_{0}^{T}\left|x^{\prime}(t)\right| d t \\
\leq & \left(r_{3}+\varepsilon\right) \int_{0}^{T}|x(t-\tau(t))|\left|x^{\prime}(t)\right| d t+\left(\|e\|_{\infty}+h_{1}+h_{2}\right) \int_{0}^{T}\left|x^{\prime}(t)\right| d t .
\end{aligned}
$$


Case 1. (3.11) holds. It follows from (3.17) and Hölder inequality that

$$
\begin{aligned}
& \left(r_{1}-\varepsilon\right)\left\|x^{\prime}\right\|_{2}^{2} \leq\left(r_{3}+\varepsilon\right)\|x\|_{\infty} \sqrt{T}\left\|x^{\prime}\right\|_{2}+\left(\|e\|_{\infty}+h_{1}+h_{2}\right) \sqrt{T}\left\|x^{\prime}\right\|_{2} \\
& \leq\left(r_{3}+\varepsilon\right)\left(\frac{1}{2} \sqrt{T}\left\|x^{\prime}\right\|_{2}+d\right) \sqrt{T}\left\|x^{\prime}\right\|_{2}+\left(\|e\|_{\infty}+h_{1}+h_{2}\right) \sqrt{T}\left\|x^{\prime}\right\|_{2} \\
& =\left(r_{3}+\varepsilon\right) \frac{T}{2}\left\|x^{\prime}\right\|_{2}^{2}+\left(\left(r_{3}+\varepsilon\right) d+\|e\|_{\infty}+h_{1}+h_{2}\right) \sqrt{T}\left\|x^{\prime}\right\|_{2} .
\end{aligned}
$$

From (3.9) and (3.11), we obtain that there exists a positive constant $M_{1}$ such that

$$
\left\|x^{\prime}\right\|_{2} \leq M_{1}, \quad \text { and } \quad\|x\|_{\infty} \leq M_{1} .
$$

Case 2. (3.12) holds. It follows from (3.17), Lemma 2.2 and Hölder inequality that

$$
\begin{aligned}
\left(r_{1}-\varepsilon\right)\left\|x^{\prime}\right\|_{2}^{2} \leq & \left(r_{3}+\varepsilon\right) \int_{0}^{T}|x(t)-x(t-\tau(t))|\left|x^{\prime}(t)\right| d t+\left(r_{3}+\varepsilon\right) \int_{0}^{T}|x(t)|\left|x^{\prime}(t)\right| d t \\
& +\left(\|e\|_{\infty}+h_{1}+h_{2}\right) \int_{0}^{T}\left|x^{\prime}(t)\right| d t \\
\leq & \left(r_{3}+\varepsilon\right)\left(\int_{0}^{T}|x(t)-x(t-\tau(t))|^{2} d t\right)^{\frac{1}{2}}\left(\int_{0}^{T}\left|x^{\prime}(t)\right|^{2} d t\right)^{\frac{1}{2}} \\
& +\left(r_{3}+\varepsilon\right)\left(\int_{0}^{T}|x(t)|^{2} d t\right)^{\frac{1}{2}}\left(\int_{0}^{T}\left|x^{\prime}(t)\right|^{2} d t\right)^{\frac{1}{2}} \\
& +\left(\|e\|_{\infty}+h_{1}+h_{2}\right) \int_{0}^{T}\left|x^{\prime}(t)\right| d t \\
\leq & \left(r_{3}+\varepsilon\right)\left(\alpha \int_{0}^{T}\left|x^{\prime}(t)\right|^{2} d t\right)^{\frac{1}{2}}\left(\int_{0}^{T}\left|x^{\prime}(t)\right|^{2} d t\right)^{\frac{1}{2}} \\
& +\left(r_{3}+\varepsilon\right)\left(\frac{T}{\pi}\left\|x^{\prime}\right\|_{2}+d \sqrt{T}\right)\left\|x^{\prime}\right\|_{2}+\left(\|e\|_{\infty}+h_{1}+h_{2}\right) \sqrt{T} \|_{x^{\prime} \|_{2}} \\
= & \left(r_{3}+\varepsilon\right)\left(\alpha+\frac{T}{\pi}\right)\left\|x^{\prime}\right\|_{2}^{2}+\left(\left(r_{3}+\varepsilon\right) d+\|e\|_{\infty}+h_{1}+h_{2}\right) \sqrt{T}\left\|x^{\prime}\right\|_{2} .
\end{aligned}
$$

From (3.9) and (3.12), we know there exists a positive constant $M_{2}$ such that

$$
\left\|x^{\prime}\right\|_{2} \leq M_{2}, \quad \text { and } \quad\|x\|_{\infty} \leq M_{2}
$$

Take $R_{1}=\max \left\{M_{1}, M_{2}\right\}$. Then, if (3.1) holds, we have

$$
\left\|x^{\prime}\right\|_{2} \leq R_{1}, \quad \text { and } \quad\|x\|_{\infty} \leq R_{1}
$$

By the first equation of (3.3), we have

$$
\int_{0}^{T} \frac{y(t)}{\sqrt{1-y^{2}(t)}} d t=0 .
$$


Then there exists $\eta \in[0, T]$ such that $y(\eta)=0$. It implies that

$$
y(t)=\int_{\eta}^{t} y^{\prime}(s) d s+y(\eta)=\int_{\eta}^{t} y^{\prime}(s) d s
$$

and

$$
\|y\|_{\infty} \leq \int_{0}^{T}\left|y^{\prime}(s)\right| d s
$$

From the second equation of (3.3), we get

$$
\int_{0}^{T}\left|y^{\prime}(s)\right| d s \leq \lambda \int_{0}^{T}\left|f\left(t, \frac{1}{\lambda} x^{\prime}(t)\right)\right| d t+\lambda \int_{0}^{T}|g(t, x(t-\tau(t)))| d t+\lambda \int_{0}^{T}|e(t)| d t .
$$

Noticing that $\|x\|_{\infty} \leq R_{1}$, we have there exists $k>0$, such that

$$
|g(t, x(t-\tau(t)))| \leq k, \quad \forall t \in[0, T] .
$$

Then, from (3.13), we have

$$
\begin{aligned}
\int_{0}^{T}\left|y^{\prime}(t)\right| d t & \leq\left(r_{2}+\varepsilon\right) \int_{0}^{T}\left|x^{\prime}(t)\right| d t+\lambda \int_{0}^{T} h_{1} d t+\lambda \int_{0}^{T} k d t+\lambda \int_{0}^{T}|e(t)| d t \\
& \leq\left(r_{2}+\varepsilon\right) \sqrt{T}\left\|x^{\prime}\right\|_{2}+\left(h_{1}+k+\|e\|_{\infty}\right) T \\
& \leq\left(r_{2}+\varepsilon\right) \sqrt{T} R_{1}+\left(h_{1}+k+\|e\|_{\infty}\right) T:=R_{2} .
\end{aligned}
$$

Hence, $\|y\|_{\infty} \leq R_{2}$.

Let $\Omega_{2}=\{z \in \operatorname{ker} L: N z \in \operatorname{Im} L\}$. If $z \in \Omega_{2}$, then $z \in \operatorname{ker} L$ and $Q N z=0$. Obviously,

$$
|x(t)| \leq R_{1}, \quad y(t)=0 \leq R_{2} .
$$

Set

$$
\Omega=\left\{z=(x, y)^{\top} \in X:\|x\|_{\infty}<R_{1}+1,\|y\|_{\infty}<R_{2}+1\right\},
$$

then (1) and (2) of Lemma 2.1 are satisfied.

Next, we claim that (3) of Lemma 2.1 is also satisfied. For this, we define the isomorphism $J: \operatorname{Im} Q \rightarrow \operatorname{ker} L$ by

$$
J(x, y)=(-y, x),
$$

and let $H(\nu, \mu)=\mu \nu+(1-\mu) J Q N v, \forall(v, \mu) \in \Omega \times[0, T]$.

By simple calculations, we obtain, for $(z, \mu) \in \partial(\Omega \cap \operatorname{ker} L) \times[0,1]$,

$$
z^{\top} H(z, \mu)=\mu\left(x^{2}+y^{2}\right)+\frac{1-\mu}{T} x \int_{0}^{T}(g(t, x(t-\tau(t)))-e(t)) d t .
$$

Obviously, it follows from (H2) that $z^{\top} H(z, \mu)>0$. 
Then

$$
\begin{aligned}
\operatorname{deg}(J Q N, \Omega \cap \operatorname{ker} L, 0) & =\operatorname{deg}(H(z, 0), \Omega \cap \operatorname{ker} L, 0) \\
& =\operatorname{deg}(H(z, 1), \Omega \cap \operatorname{ker} L, 0) \\
& =\operatorname{deg}(I, \Omega \cap \operatorname{ker} L, 0) \neq 0,
\end{aligned}
$$

which implies condition (3) of Lemma 2.1 is also satisfied.

Thus $L z=N z$ has a solution $z=(x(t), y(t))^{\top}$, i.e., Equation (1.1) has a $T$-periodic solution $x(t)$ with $\|x\|_{\infty} \leq R_{1}$. This completes the proof.

Remark 3.1 If $\frac{r_{3}}{r_{1}}<\frac{2}{T}$, the condition (H4) can be not assumed, i.e., it follows only from (H1)-(H3) that Equation (1.1) has a $T$-periodic solution.

\section{An example}

In this section, as applications for Theorem 3.1, we list the following example.

Example 4.1 Consider prescribed mean curvature Rayleigh equation with a deviating argument

$$
\left(\frac{x^{\prime}}{\sqrt{1+x^{\prime 2}}}\right)^{\prime}+f\left(t, x^{\prime}(t)\right)+g\left(t, x\left(t-\frac{1}{2} \cos ^{2} t\right)\right)=\cos t
$$

where $f(t, x)=\left(2+\frac{1}{2} \sin ^{2} t\right) \frac{x^{3}}{\sqrt{1+x^{4}}}, g(t, x)=\frac{1}{3}\left(1+\sin ^{4} t\right) \frac{x^{5}}{\sqrt{1+x^{8}}}$.

Let $T=2 \pi$. Clearly, $r_{1}=2, r_{2}=\frac{3}{2}, r_{3}=\frac{2}{3}, \alpha=\frac{1}{2}$, and

$$
\frac{r_{3}}{r_{1}}=\frac{1}{3}<\max \left\{\frac{2}{2 \pi}, \frac{1}{\frac{1}{2}+\frac{2 \pi}{\pi}}\right\}=\frac{2}{5} .
$$

From Theorem 3.1, Equation (4.1) has at least one $T$-periodic solution.

Remark 4.1 If $f(t, x)=\left(2+\frac{1}{2} \sin ^{2} t\right) x, g(t, x)=\frac{1}{3}\left(1+\sin ^{4} t\right) x$, Equation (4.1) is a prescribed mean curvature Liénard equation. By using Theorem 3.1 , it has at least one $2 \pi$-periodic solution, which cannot be obtained by [15]. This implies that the results of this paper are essentially new.

\section{Competing interests}

The authors declare that they have no competing interests.

\section{Authors' contributions}

All authors have equally contributed in obtaining new results in this article and also read and approved the final manuscript.

\section{Acknowledgements}

Research supported by National Science foundation of China, No. 10771145 and the Beijing Natural Science Foundation (Existence and multiplicity of periodic solutions in nonlinear oscillations), No. 1112006. 


\section{References}

1. Cheung, W, Ren, J: Periodic solutions for $p$-Laplacian Liénard equation with a deviating argument. Nonlinear Anal. 59 107-120 (2004)

2. Cheung, W, Ren, J: On the existence of periodic solutions for $p$-Laplacian generalized Liénard equation. Nonlinear Anal. 60, 65-75 (2005)

3. Lu, S, Ge, W: Periodic solutions for a kind of Liénard equations with deviating arguments. J. Math. Anal. Appl. 249 231-243 (2004)

4. Lu, S, Ge, W: Sufficient conditions for the existence of periodic solutions to some second order differential equations with a deviating argument. J. Math. Anal. Appl. 308, 393-419 (2005)

5. Lu, S, Gui, Z: On the existence of periodic solutions to $p$-Laplacian Rayleigh differential equation with a delay. J. Math. Anal. Appl. 325, 685-702 (2007)

6. Zong, M, Liang, $\mathrm{H}$ : Periodic solutions for Rayleigh type $p$-Laplacian equation with deviating arguments. Appl. Math. Lett. 20,43-47 (2007)

7. Gao, H, Liu, B: Existence and uniqueness of periodic solutions for forced Rayleigh-type equations. Appl. Math. Comput. 211, 148-154 (2009)

8. Ma, T, Wang, Z: A continuation lemma and its applications to periodic solutions of Rayleigh differential equations with subquadratic potential conditions. J. Math. Anal. Appl. 385, 1107-1118 (2012)

9. Yu, Y, Shao, J, Yue, G: Existence and uniqueness of anti-periodic solutions for a kind of Rayleigh equation with two deviating arguments. Nonlinear Anal. 71, 4689-4695 (2009)

10. Lv, X, Yan, P, Liu, D: Anti-periodic solutions for a class of nonlinear second-order Rayleigh equations with delays. Commun. Nonlinear Sci. Numer. Simul. 15, 3593-3598 (2010)

11. Liu, B: Anti-periodic solutions for forced Rayleigh-type equations. Nonlinear Anal., Real World Appl. 10, $2850-2856$ (2009)

12. Bonheure, D, Habets, P, Obersnel, F, Omari, P: Classical and non-classical solutions of a prescribed curvature equation J. Differ. Equ. 243, 208-237 (2007)

13. Pan, $\mathrm{H}$ : One-dimensional prescribed mean curvature equation with exponential nonlinearity. Nonlinear Anal. 70 , 999-1010 (2009)

14. Benevieria, P, Do Ó, JM, Medeiros, ES: Periodic solutions for nonlinear systems with mean curvature-like operators. Nonlinear Anal. 65, 1462-1475 (2006)

15. Feng, M: Periodic solutions for prescribed mean curvature Liénard equation with a deviating argument. Nonlinear Anal., Real World Appl. 13, 1216-1223 (2012). doi:10.1016/j.nonrwa.2011.09.015

16. Gaines, RE, Mawhin, J: Coincidence Degree and Nonlinear Differential Equations. Lecture Notes in Mathematics, vol. 568. Springer, Berlin (1977)

17. Lu, S: Homoclinic solutions for a class of second-order $p$-Laplacian differential systems with delay. Nonlinear Anal., Real World Appl. 12, 780-788 (2011)

18. Mawhin, J, Willem, M: Critical Point Theory and Hamiltonian Systems. Application of Mathematical Science, vol. 74. Springer, New York (1989)

doi:10.1186/1687-1847-2013-88

Cite this article as: Li et al.: Periodic solutions for prescribed mean curvature Rayleigh equation with a deviating argument. Advances in Difference Equations 2013 2013:88.

\section{Submit your manuscript to a SpringerOpen ${ }^{\circ}$ journal and benefit from:}

- Convenient online submission

Rigorous peer review

Immediate publication on acceptance

- Open access: articles freely available online

- High visibility within the field

- Retaining the copyright to your article 